\title{
Quark and Gluon Jets in the Breit Frame of Lepton-Nucleon Scattering *
}

\author{
K.H. Streng \\ Institut $f$. Theoretische Physik. TH Aachen, D-5100 Aachen, Federal Republic of Germany \\ T.F. Walsh \\ Deutsches Elektronen-Synchrotron DESY, D-2000 Hamburg, Federal Republic of Germany \\ P.M. Zerwas \\ Institut f. Theoretische Physik. TH Aachen, D-Federal Republic of Germany
}

Received 27 March 1979

\begin{abstract}
Gluon bremsstrahlung, $q \rightarrow G q$, and quark pair production from gluons, $G \rightarrow q \bar{q}$, in deep inelastic reactions is investigated in the Breit frame (moving along $\mathbf{Q}$ in the laboratory). These QCD effects diminish the overall forward momentum. There are also events with a single large $p_{\perp}$ forward jet. One spectacular class of events is predicted in which no forward going hadrons emerge in the Breit frame. These effects are not mimicked by nonperturbative (limited $p_{\perp}$ ) parton jets at large but attainable $Q^{2}$.
\end{abstract}

\section{Introduction}

Evidence for quantum chromodynamics (QCD) [1] as the basis for the field theoretic description of strong interactions is steadily growing [2]. A direct proof in high energy experiments for the existence of the building blocks of the theory, quarks and gluons, does not (yet) exist. To the contrary, there is a theoretical suspicion that these colored objects might not be observable as free particles (at least in the present energy range). The most direct evidence for their existence is expected to come from the observation of hadron jets, generated after quarks and gluons are accelerated (e.g. when quarks absorb a virtual photon in electroproduction processes). Whereas quark jets can readily be studied in $e^{+} e^{-}$ annihilation and all deep inelastic lepton-nucleon reactions, it is experimentally much harder to investigate gluon jets. The most detailed analysis so far has been carried out by the Pluto collaboration at DORIS [3], which studied the evidence for gluon

\footnotetext{
* Supported in part by the W. German Bundesministerium für
} Forschung und Technologie jets among the decay products of the heavy quarkantiquark $Y$ resonance.

It was originally suggested that one look for gluons as bremsstrahlung quanta (developing into jets) off quarks in $e^{+} e^{-}$annihilation and deep inelastic lepton-nucleon scattering [4]. In this paper we pursue some experimental consequences of gluon bremsstrahlung in lepton-quark collisions. We point out that investigating this process in the Breit frame (connected to the lab frame by a Lorentz boost along the direction of the momentum transfer vector) provides experimental signals which clearly separate this process from the otherwise dominating zeroth order parton mechanism. The key point is that in the naive parton model the struck quark moves forward in the Breit frame (along Q) carrying a momentum $\sqrt{Q^{2}} / 2$ independent of $x$ [5]. Neglecting hadron masses and transverse momenta for large $Q^{2}$, we find precisely this momentum in the quark fragments, i.e. in those hadrons which move forward. in the Breit frame. Allowing for gluon radiation without a transverse momentum cut off changes this picture drastically. (i) The average longitudinal momentum of the forward going hadrons is reduced to a fraction of $Q / 2$. For large enough $Q^{2}$ this effect is much stronger than the finite mass and transverse momentum effect in the parton jet. (ii) In some events the total transverse momentum of the forward going hadrons in the Breit frame is not zero. It is balanced, of course, by large transverse momentum hadrons moving backwards in this frame. (iii) There are even a few events predicted in which no hadron is moving forward, a rather spectacular signature. It is for forward moving hadrons that we expect the clearest analogy to the structure of final states in $e^{+} e^{-}$collisions. (There is of course a great convenience in having an axis selected by $\mathbf{Q}$ in $l N$ compared 
to $e^{+} e^{-}$where no a priori jet axis exists.) The backward jet or jets in the Breit frame, however, are novel to deep inelastic reactions, as they contain target remnants.

Section II describes the kinematics of gluon bremsstrahlung and quark pair production out of gluons in the Breit frame. In addition, we discuss the main features of the bremsstrahlung and pair production cross sections. In Section III we present our numerical results for the momentum distributions of the forward going hadrons in the Breit frame, the number of events with unbalanced transverse momentum in this region and related subjects.

\section{Breit Frame Analysis of QCD Effects}

We are interested in hadron production in deep inelastic lepton-nucleon scattering

$l+N \rightarrow l^{\prime}+$ hadrons .

The momentum transfer involved is $\left(k_{l}-k_{l}\right)^{2}=-Q^{2}$ and the Bjorken variable $x=Q^{2} / 2 P_{N} \cdot q$. We choose $\mathbf{Q}$ as the (positive) $z$-axis of the various reference frames we consider. The transformation from the laboratory frame to the Breit frame is reached by a Lorentz boost along the direction of the momentum transfer vector. The boost is given by $\gamma v=$ $-Q /(2 M x) ;$ the only nonvanishing component of the momentum transfer vector in this Breit frame is the $z$-component, $q_{B}=(0,0,0, Q)$. Hadrons which move forward in the Breit frame must have a component of the 3-momentum parallel to the momentum transfer vector in the laboratory frame fulfilling the requirement

$p_{L}^{\|} \geqq Q m_{\perp} /(2 M x)$

where $m_{\perp}$ is the transverse mass $\left(m^{2}+\mathbf{p}_{1}^{2}\right)^{1 / 2}$. In the following we will mainly be interested in the distribution of these forward moving secondaries. They belong to quark and gluon jets for which clear theoretical conjectures have been developed [5]. Backward moving particles in the Breit frame belong to the current and hadron plateaus or the target and hole fragmentation regions [6] about which little is known so far (Fig. 1.)

Deep inelastic lepton-nucleon scattering is described at small distances in the parton model (zeroth order QCD) by the diagrams in Fig. 2. For massless partons, carrying no transverse momentum, the momentum of the struck quark in the final state is $+Q / 2$ in the Breit frame. In the limit where masses and transverse momenta of the hadrons in the resulting quark jet can be neglected, this is also the momentum of all the particles in the jet. Defining the scaling variable

$z_{\|}=2 \sum_{i} p_{B}^{\|}(i) / Q$

where the sum runs over all forward moving hadrons

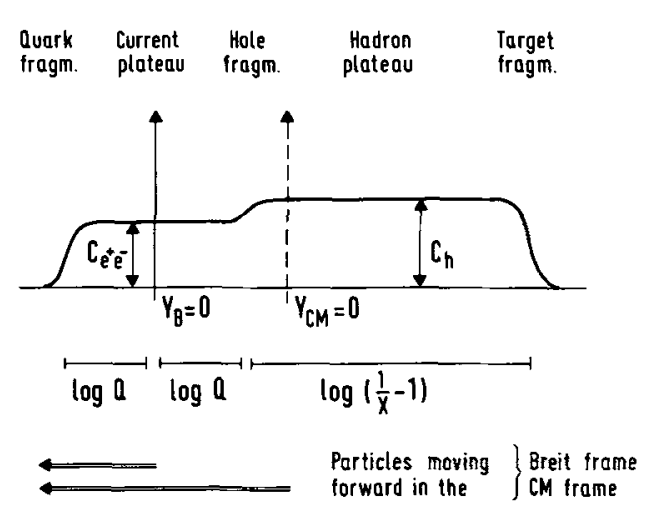

Fig. 1. Rapidity distributions of final hadrons in deep inelastic lepton-nucleon scattering. $y=0$ is shown for the Breit frame (full line) and the center-of mass frame of the hadrons (broken line)

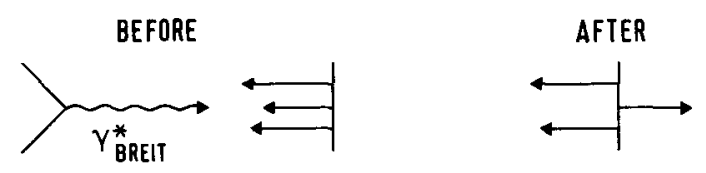

Fig. 2. Parton configurations before and after the absorption of the virtual photon

in the Breit frame, we obtain for $Q \rightarrow \infty$.

$\frac{d \sigma^{\text {Parton }}}{d x d Q^{2} d z_{\|}} / \frac{d \sigma}{d x d Q^{2}}=\delta\left(z_{\|}-1\right)$,

similar to the thrust distribution in the parton model of high energy $e^{+} e^{-}$annihilation. In the next chapter we will describe how this peak is smeared out by masses and transverse momenta of the hadrons in the jet for large but finite $Q$. On dimensional grounds we expect a spread of the order of $\left\langle\Delta z_{\|}\right\rangle \sim$ $2\left\langle p_{\perp}\right\rangle \log Q / Q \sim 1 / Q(\mathrm{GeV})$.

First order QCD corrections to the parton model reaction $\gamma^{*}+q \rightarrow q\left(\gamma^{*}\right.$ stands for the absorbed virtual photon in electroproduction, etc.) change this picture dramatically. In this order, the struck quark can radiate a hard gluon bremsstrahlung quantum

$\gamma^{*}+q \rightarrow q+G$,

and quark pair production can occur in collisions of the photon with gluonic partons

$\gamma^{*}+G \rightarrow q+\bar{q}$

Since the transverse momentum is not bounded, the final state will consist of three jets: two from the gauge quanta in (5a), (5b) and a third "target" jet. We define $x_{P}$ as the Bjorken variable for a parton target (momentum $p_{P}$ ),

$x_{P}=Q^{2} / 2\left(q p_{P}\right) \quad$ with $x \leqq x_{P} \leqq 1$,

and $z_{P}$ as the corresponding Feynman scaling variable 


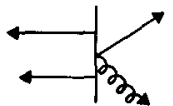

A
B

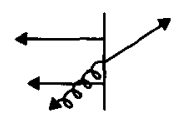

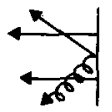

C
Fig. 3. Quark and gluon configurations after the absorption of the virtual photon in the Breit frame

(the momentum of the final state quark/gluon is $\left.p_{P}^{\prime}\right)^{1}$

$z_{P}=\left(p_{P}^{\prime} p_{P}\right) /\left(q p_{P}\right)$.

$x_{P}$ and $z_{P}$ determine the longitudinal and transverse momentum of the final state quark and gluons (and their respective jets) in the Breit frame. In units of $Q / 2$,

$z_{\|}=\frac{1}{x_{P}}\left[x_{P}+z_{P}-1\right]$

$z_{\perp}=2 \sqrt{\frac{z_{p}}{x_{P}}} \cdot \sqrt{\left(1-x_{P}\right)\left(1-z_{P}\right)}$.

The longitudinal momentum of the accompanying parton in the final state is found by replacing $z_{P} \Rightarrow$ $1-z_{P}$, giving

$\bar{z}_{\|}=\frac{1}{x_{P}} \cdot\left[x_{P}-z_{P}\right]$;

the absolute value of the transverse momentum is of course the same for both partons.

Three different event types are found, depending on the signs of $z_{\|}$and $\bar{z}_{\|}$which can be positive and negative.

(A) $z_{\|}$and $\bar{z}_{\|}>0$ :

two jets are moving forward in the Breit frame and the forward going particles balance transverse momentum relative to the $\mathbf{Q}$ axis (Fig. 3a).

(B) $z_{\|}>0$ and $\bar{z}_{\|}<0$ or vice versa:

only one jet goes forward in the Breit frame leaving transverse momentum unbalanced in this region (Fig. 3b); the second jet is found moving opposite to $\mathbf{Q}$, yet with large transverse momentum.

(C) $z_{\|}$and $\bar{z}_{\|}<0$ :

the forward direction of the Breit frame remains empty and both jets are running backwards (Fig. 3c) ${ }^{2}$

The $\left[x_{p}, z_{p}\right]$ regions corresponding to the above configurations are the triangles shown in Fig. 4.

Méndez [7] has tabulated the jet cross sections

\footnotetext{
1 Since $p_{P} \sim$ nucleon momentum, $z_{P}$ is the fraction of the hadron energy in the laboratory frame which is found in the final state quark/gluon

${ }^{2}$ This configuration is not in conflict with longitudinal momentum conservation; due to the large transverse momentum the sign of the longitudinal momentum of the jets need not be conserved when boosting from the Breit frame to the CM frame
}

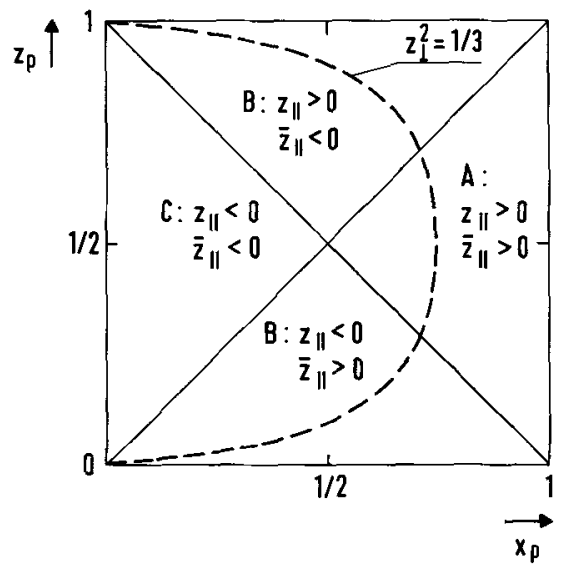

Fig. 4. $\left[x_{p}, z_{p}\right]$ areas of the configurations $A, B$ and $C$ in Fig. 3 . Jet events with fixed $z_{\perp}$ are located on the dotted line

and we adopt here his results for muo-production ${ }^{3}$

$$
\begin{aligned}
& \frac{d \sigma(\mu N)^{\mathrm{QCD}}}{d x d Q^{2} d x_{P} \overline{d z_{P}}} \\
& =\frac{\alpha^{2} \cdot \alpha_{s}\left(Q^{2}\right)}{Q^{4}} x_{P}^{-1}\left[\sum_{q, \bar{q}} e_{q}^{2} f_{q}\left(\frac{x}{x_{P}}\right) A_{q}\left(x_{P}, z_{P}, y\right)\right. \\
& \left.+\sum_{q} e_{q}^{2} f_{G}\left(\frac{x}{x_{P}}\right) A_{G}\left(x_{P}, z_{P}, y\right)\right]
\end{aligned}
$$

with $\alpha_{s}$ denoting the running QCD coupling constant; $f_{q, G}$ the quark and gluon densities in the nucleon; and

$$
\begin{aligned}
& A_{q}\left(x_{P}, z_{P}, y\right)=\frac{32}{3}[1-y] x_{P}+\frac{4}{3}\left[1+(1-y)^{2}\right] \\
& \cdot\left[\left(1-x_{P}\right)+\frac{1+x_{P}^{2}\left[z_{P}^{3}+\left(1-z_{P}\right)^{3}\right]}{\left(1-x_{P}\right) z_{P}\left(1-z_{P}\right)}\right] \\
& A_{G}\left(x_{p}, z_{p}, y\right)=16[1-y] x_{P}\left(1-x_{P}\right) \\
& +\left[1+(1-y)^{2}\right]\left[x_{P}^{2}+\left(1-x_{P}\right)^{2}\right] \frac{z_{P}^{2}+\left(1-z_{P}\right)^{2}}{z_{P}\left(1-z_{P}\right)}
\end{aligned}
$$

The zeroth order parton cross section is well known to be

$\frac{d \sigma(\mu N)}{d x \bar{d} Q^{2}}=\frac{2 \pi \alpha^{2}}{Q^{4}}\left[1+(1-y)^{2}\right] \sum_{q: \bar{q}} e_{q}^{2} f_{q}(x)$.

Armed with this, we can proceed to make some numerical estimates for the effects described previously.

\section{Numerical Estimates}

Being interested in qualitative estimates only we use the following simple parametrizations of quark

\footnotetext{
${ }^{3} z_{p}$ can be measured event by event as the fraction of the total hadron energy in the jet (lab frame, see (6)), $x_{P}$ is determined by the transverse momentum of the jet: $x_{p}=\left[1+z_{\perp}^{2} / 4 z_{P}\left(1-z_{P}\right)\right]^{-1}$ from (7)
} 


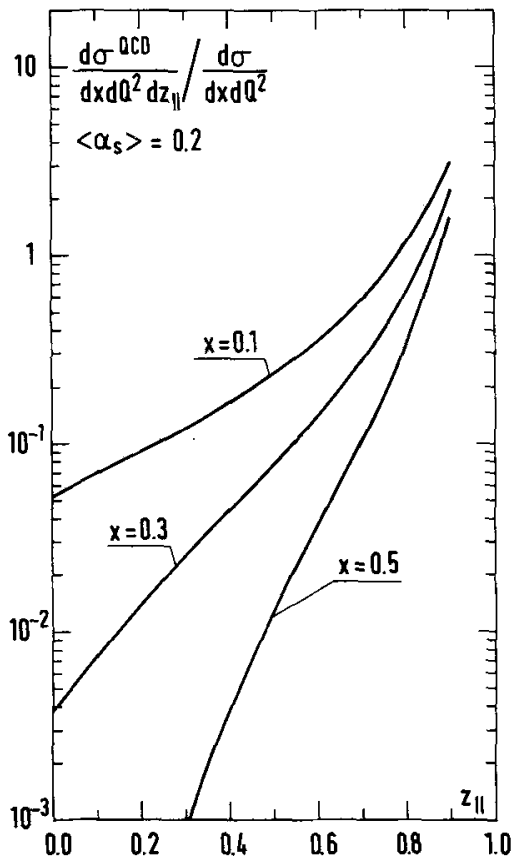

Fig. 5. $z_{11}$ distribution of quark and gluon jets for three values of Bjorken $x$ and $y=0$. The distribution depends (via the strong coupling constant) only logarithmically on $Q^{2}$

and gluon densities in the (isoscalar) nucleon [8]:

$$
\begin{aligned}
& \sum_{q, \bar{q}} e_{q}^{2} f_{q}(\xi)=\xi^{-1} F_{2}(\xi) \simeq 1.31(1-\xi)^{3}+.86(1-\xi)^{4} \\
& +37 \xi^{-1 / 2}(1-\xi)^{5}+.21 \xi^{-1}(1-\xi)^{7} \\
& \sum_{q} e_{q}^{2} f_{G}(\xi) \simeq \frac{2}{3} f_{G}(\xi) \sim \frac{5}{3} \xi^{-1}(1-\xi)^{4}
\end{aligned}
$$

For the running coupling constant we assume an average value of $\alpha_{\mathrm{s}}=0.2$ corresponding to $Q$ in the $10 \mathrm{GeV}$ range. The dependence on $y=Q^{2} / 2 M E x$ will generally not be discussed since this parameter does not affect the results in a crucial way (except for antineutrino scattering); if not stated otherwise we put $y=0$.

\section{A) Longitudinal Momentum Distribution of Jets}

In zeroth order QCD the longitudinal momentum of all forward going hadrons in the Breit frame is just $Q / 2$ if masses and transverse momenta are neglected, i.e. $z_{\|}=1$. Including gluon bremsstrahlung and pair production in first order QCD we obtain the $z_{\|}$distribution shown in Fig. $5\left(z_{\|}=2 \sum_{i} p_{B}^{\|}(i) / Q\right.$ with $p_{B}^{\|}(i)=$ momentum of hadron $i$ in the Breit frame $\geqq 0$ ). For $z_{\|} \rightarrow 1$ the emitted gluons are soft or parallel to $Q$ and the perturbative approach must break down.

Before the QCD result can be used one must estimate the spread in the parton result due to the finite jet mass. It is more difficult to solve this problem in deep inelastic scattering than $e^{+} e^{-}$annihilation

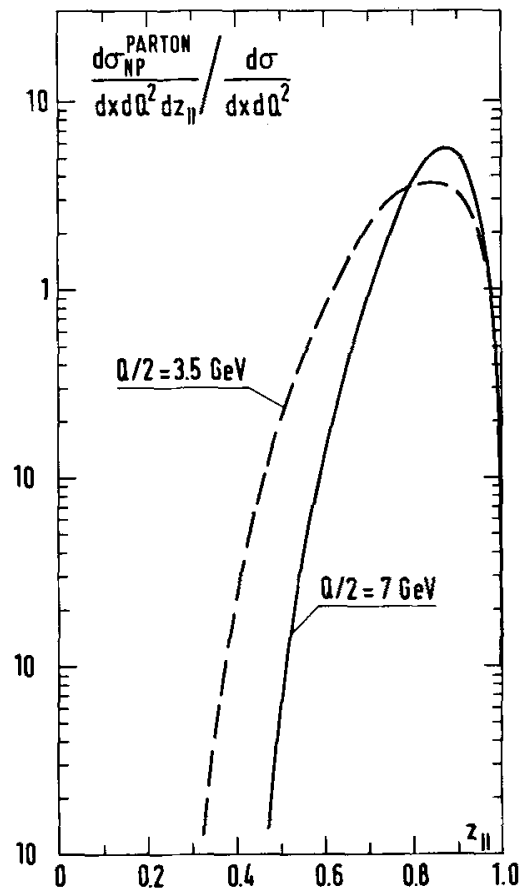

Fig. 6. Estimated lower limit of the nonperturbative spread of a forward moving jet (Fig. 2) towards zero in the Breit frame for two different values of the momentum transfer.

since the parton configurations in the current and target fragmentation region are different. Therefore, the hadron distributions differ as well. In order to facilitate the solution of this problem we restrict ourselves to estimating (what we expect to be) a lower limit for the nonperturbative spread in $z_{\|}(\rightarrow 0)$ of the forward moving jet in the Breit frame. The model is defined as follows. Energy-momentum conservation for the absorption of a virtual photon by a parton, leading to a jet, gives an inequality for the energy of this jet in the Breit frame, $E_{J} \geqq Q / 2$. Therefore assuming the jet energy to be $Q / 2$ we expect to underestimate its $z_{\|}$distribution. Figure 6 presents this estimate for $Q / 2=3.5$ and $7 \mathrm{GeV}$, where the particle distribution within the jet is generated according to the prescription of Field and Feynman [9]. Comparing the nonperturbative with the perturbative $z_{\|}$distribution we see that a surplus of low $z_{\|}$jets is predicted by QCD. This is just like the QCD prediction of low thrust events in $e^{+} e^{-}$annihilation.

We note again that the nonperturbative jet moves rapidly toward $z_{\| \mid}=1$ as $Q^{2}$ increases. Thus $d \sigma_{\mathrm{NP}}^{\text {parton }}$ decreases rapidly with $Q^{2}$ at fixed $x$ and $z_{\|}$. On the other hand, $d \sigma$ QCD only decreases logarithmically with $Q^{2}$. Plots of data as in Figs. 5 and 6 at different $Q^{2}$ should expose the two types of contributions.

\section{B) Unbalanced Particle Jets}

Hadron distributions of quark jets are rotationally 


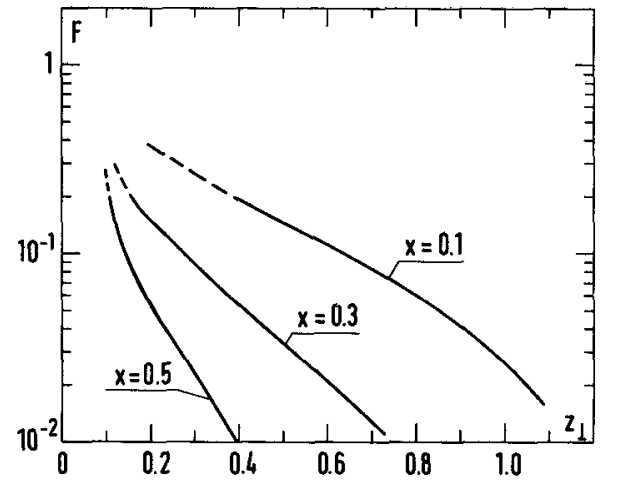

Fig. 7. Fraction of events in which one jet is moving forward in the Breit frame and carrying a transverse momentum $p_{\perp}^{\text {Jet }} \geqq z_{\perp} Q / 2$

invariant with respect to the jet axis and the total transverse momentum of the particles in the jet sums up to zero. Within an uncertainty of $\sim \frac{1}{2} \mathrm{GeV}$, the average transverse momentum of the quarks in the nucleon, this is also true for the hadron distribution around the momentum transfer axis in the parton model. Gluon bremsstrahlung and quark pair production however lead in region $\mathrm{B}$ of the $\left[x_{p}, z_{P}\right]$ square, to one forward going jet in the Breit frame the transverse momentum of which grows with $Q$ and is not balanced by a second forward moving jet. Figure 7 shows the relative number of unbalanced jet events with a transverse momentum larger than a fraction $z_{\perp}$ of $Q / 2$. The magnitude of the effect is encouraging particularly because we have to compare it with a null-effect in the naive parton model.

\section{C) No Particles in the Forward Cone}

Finally we mention the amusing fact that in a small fraction of events no particles are moving forward in the Breit frame. This fraction is infrared finite. The $x_{P}$ and $z_{P}$ values of those events are located in region C of Fig. 4. The rate is shown in Fig. 8 as a function of $x$.

The procedure used in this section can be expanded to calculate other quantities of interest. One example is the fraction of the total forward energy outside a cone of half angle $\delta$ [10]. (There are actually two such cones: one directed along $\mathbf{Q}$ and one along $-\mathbf{Q}$. The energy fraction is then that between angle $\delta$ and $\pi / 2$.)

\section{Summary}

Experimental proof of the existence of gluons is one of the most important issues of high energy $e^{+} e^{-}$annihilation and deep inelastic lepton-nucleon scattering. Assuming that gluons reveal themselves as jets we have shown in the preceding paragraphs that the Breit frame analysis of deep inelastic leptonnucleon scattering is expected to provide clean
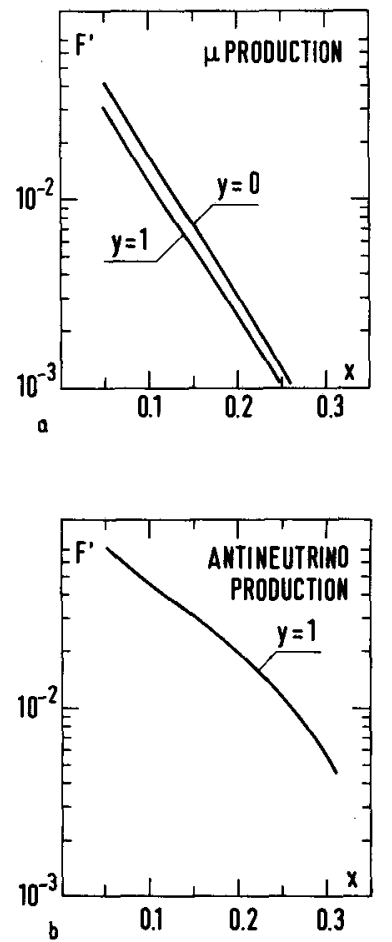

Fig. 8a and b. Fraction of events with no jet going forward in the Breit frame, a muon-nucleon scattering; $b$ antineutrino-nucleon scattering at $y=1$

signatures of hard gluon bremsstrahlung and quark pair production out of gluons. Describing QCD phenomena in this frame is most closely related to the centre-of-mass-frame in $e^{+} e^{-}$annihilation. The momentum transfer $\sqrt{Q^{2}}$ in deep inelastic scattering corresponds directly to the centre-of-massenergy $\sqrt{s}$ in $e^{+} e^{-}$. This is exemplified here for muo-production, but the same applies to neutrinoproduction as well. In particular, antineutrino reactions should be most powerful in revealing first order QCD effects [11]. For $y \rightarrow 1$ the zeroth order (finite $p_{\perp}$ ) parton contribution to the cross section is strongly suppressed by helicity and the small antiquark content of the nucleon. By contrast, the first order QCD contribution has only a weak $y$ dependence. Thus the QCD effects become more visible as $y \rightarrow 1$. It will be very interesting to confront the QCD predictions to data in deep inelastic muon and neutrino reactions.

Acknowledgement. We are indebted to H.G. Sander for computing the nonperturbative spread of the quark jet, displayed in Fig. 6.

\section{References}

1. Fritzsch, H., Gell-Mann, M., Leutwyler, H. : Phys. Lett. B47, 365 (1973)

2. Anderson, H.L. et al.: Phys. Rev. Lett. 40, 1061 (1978); 
Bosetti, P.C. at al. : Nucl. Phys. B142, 1 (1978); de Groot, J.G.H. et al. : CERN preprints, 1978

3. Berger, C. et al. : DESY preprint $78 / 71$

4. Ellis, J., Gaillard, M.K., Ross, G.G. : Nucl. Phys. B111, 253 ; (1976) Floratos, E.G. : Nuovo Cimento 43A, 241 (1978)

5. Feynman, R.P.: Photon-Hadron Interactions, Reading: Benjamin 1972

6. Bjorken, J.D. : Phys. Rev. D7, 282 (1973); Cahn, R.N.,
Cleymans, J., Colglazier, E.W. : Phys. Lett. 43B, 323 (1973)

7. Méndez, A.: Nucl. Phys. B145, 199 (1978)

8. Wilson, W. Berkely preprint LBL-3862

9. Field, R.D., Feynman, R.P. : Nucl. Phys. B136, 1 (1978)

10. Bigi, I.I.Y., Walsh, T.F. : DESY preprint $78 / 72$

11. Köpp, G., Maciejko, R., Zerwas, P.M. : Nucl. Phys. B144, 123 (1978); Stevenson, P.M. : preprint, Imperial College, London 1978 